\title{
Genome-Targeted Drug Design: Understanding the Netropsin-DNA Interaction
}

\author{
Ya-Yin Fang*,a, Vernon R. Morris ${ }^{\mathrm{b}}$, Guy M. Lingani ${ }^{\mathrm{a}}$, Eric C. Long $^{\mathrm{c}}$ and William M. Southerland ${ }^{\mathrm{a}}$ \\ ${ }^{a}$ Department of Biochemistry \& Molecular Biology, Howard University College of Medicine, Washington, DC 20059, \\ USA \\ ${ }^{b}$ Department of Chemistry, Howard University College of Arts \& Sciences, Washington, DC 20059, USA \\ ${ }^{c}$ Department of Chemistry \& Chemical Biology, Indiana University-Purdue University Indianapolis (IUPUI), \\ Indianapolis, Indiana 46202, USA
}

\begin{abstract}
Knowledge of the sequence of the human genome has provided significant opportunities to exploit DNA as a target in the rational design of therapeutic agents. Among agents that target DNA, netropsin exhibits a strong preference for binding $\mathrm{A} / \mathrm{T}$ rich regions. In order to investigate the key factors responsible for DNA recognition and binding by netropsin, molecular dynamics simulations were carried out on a DNA-netropsin complex in which two netropsin molecules are bound to each AATT site of the 16-mer d(CTTAATTCGAATTAAG) 2 . In this complex, the two netropsins are bound to the DNA minor groove in a head-to-head orientation with the guanidinium-termini of both netropsins pointed toward the center of the DNA. Despite their identical environments, molecular dynamics simulations showed that the two netropsins exhibited differences in their respective RMS behaviors, binding energies, minor groove width fluctuations, and rotations of their structural planes. These observations suggest that DNA recognition and binding by small molecules may be governed by mechanism(s) that are much more complex than initially anticipated and may represent unexpected challenges in genome-targeted drug design.
\end{abstract}

Keywords: DNA, minor groove, netropsin, molecular dynamics simulation.

\section{INTRODUCTION}

The molecular design of sequence-selective DNA binding agents permits recognition and targeting of this biopolymer, thereby creating the possibility of gene-directed chemotherapies [1]. Considerable research has been done on the antitumor antibiotic netropsin, which is a wellcharacterized DNA minor groove binding molecule that exhibits a strong preference for A/T-rich sequences of Bform duplex DNA [2-4]. Netropsin has also provided a design paradigm for second- and third-generation DNAtargeted compounds that exhibit increasing levels of siteselectivity $[1,5,6]$. Thus far most efforts have focused on the role of the DNA target sequence as a criterion for netropsin binding to DNA, while other key factors responsible for binding remain elusive [7, 8]. Recently, a crystallized DNA-netropsin complex was reported in which two netropsin molecules were found to bind to the 16-mer d(CTTAATTCGAATTAAG) ${ }_{2}$ [9]. Unlike other crystallographic determinations of DNA-bound netropsin reported to date [2], this sequence possesses netropsin-preferred AATT binding sites flanked by two different DNA sequences (TpT and $\mathrm{CpG}$ ). Thus, the non-identical nature of these flanking sequences permitted examination of netropsin binding behavior in an asymmetric minor groove environment [9].

*Address correspondence to this author at the Department of Biochemistry \& Molecular Biology, Howard University College of Medicine, Washington, D.C. 20059, USA; Tel: 202-806-6348; Fax: 202-518-9330; E-mail: yfang@howard.edu
Consequently, this structure indicated that asymmetry of the minor groove width in the AATT region contributes to the orientation of netropsin within the minor groove (Scheme 1).

Taking advantage of this unique DNA/netropsin complex, we investigated additional factors responsible for netropsin binding to DNA using molecular dynamics simulations. Our results show that the two bound netropsins exhibit different behaviors during the simulation, including differences in their RMS deviations, binding energies, minor groove width fluctuations, and rotations of their structural planes, respectively. These observations suggest that DNA recognition and binding by small molecules may be governed by mechanism(s) that are much more complex than initially anticipated and may represent unexpected challenges in genome-targeted drug design.<smiles>CN=C(N)NCC(=O)Nc1cc(C(=O)Nc2cc(C(=O)NCCC(=N)N)n(C)c2)n(C)c1</smiles>

Scheme 1. Diagram of netropsin including the numbering scheme for the atoms referred to herein. 


\section{COMPUTATIONAL METHODS}

All molecular dynamics simulations were performed using AMBER9 on a Silicon Graphics Tezro workstation. Default settings were used for the program unless specified otherwise. Molecular dynamics simulations were performed for the netropsin/DNA complex, free DNA and free netropsin.

\section{Starting Structures}

Starting structures were modified from the crystal structure of two netropsins bound to d(CTTAATTCGAATTAAG) $)_{2}$ in a complex with the Nterminal fragment of moloney murine leukemia virus reverse transcriptase (MMLV RT) catalytic fragment $[9,10]$ as shown in Fig. (1). The PDB code for this structure is 1ZTT. For the DNA complex, the host protein (MMLV RT) was removed and the complex of netropsin bound to the DNA was investigated using molecular dynamics simulations. In the simulation system each strand of DNA had $15 \mathrm{PO}_{4}{ }^{-}$ anions and each netropsin had two positive charges. To achieve electroneutrality, $26 \mathrm{Na}^{+}$counterions were added using the addIons algorithm in the XLEaP facility of AMBER. The complex was subsequently solvated using a water box with 6019 TIP3P water molecules. The box dimensions were $74.68 \AA \times 70.86 \AA \times 49.00 \AA$ with a corresponding $\Gamma$ value (waters/nucleotide) of 234.96. For simulation of free DNA, the host protein and netropsins were removed and a total of $30 \mathrm{Na}^{+}$counterions were added as well as 6279 TIP3P waters. The box dimensions were $70.50 \AA \times 70.86 \AA \times 53.00 \AA$ with a corresponding $\Gamma$ value (waters/nucleotide) of 209.3. For the simulation of free netropsin, the host protein, DNA, and one netropsin were removed. To achieve electroneutrality in this system, $2 \mathrm{Cl}^{-}$ ions were added as well as 1608 waters. The box dimensions were $36.59 \AA \times 44.14 \AA \times 42.04 \AA$.

\section{Molecular Dynamics Simulations}

The resulting solvated netropsin-DNA complex and free DNA systems described above were subjected to five, 500step full conjugate gradient minimizations. Each minimization was carried out with harmonic restraints on DNA, counterion, and the ligand position at different levels. Initial restraints on the DNA and counter ions were $100 \mathrm{kcal}$ $(\mathrm{mol} \bullet \AA)^{-1}$. During the successive minimizations, the restraints were relaxed by $25 \mathrm{kcal}(\mathrm{mol} \bullet \AA)^{-1}$. Thus, the fifth, 500-step minimization was performed without restraints. Prior to molecular dynamics simulations, the resulting structure was heated from 100 to $300 \mathrm{~K}$ over a time period of 125 ps (with a temperature coupling of 0.2 ps and constant volume conditions) while positional restraints of $100 \mathrm{kcal}$ $(\mathrm{mol} \bullet \AA)^{-1}$ were used for the DNA and the counterions.

The resulting restraint-free systems were allowed to equilibrate for an additional $15 \mathrm{ps}$; the temperature was allowed to fluctuate around $300 \mathrm{~K}$ with a temperature coupling time of $0.2 \mathrm{ps}$ and the pressure was allowed to fluctuate around 1 bar with a pressure coupling of $0.2 \mathrm{ps}$. The equilibrated structure was used in subsequent molecular dynamics simulations. Simulations were permitted to run for $10 \mathrm{~ns}$ and coordinates were saved every $0.2 \mathrm{ps}$ for postprocessing. For the simulation of free netropsin, atomic

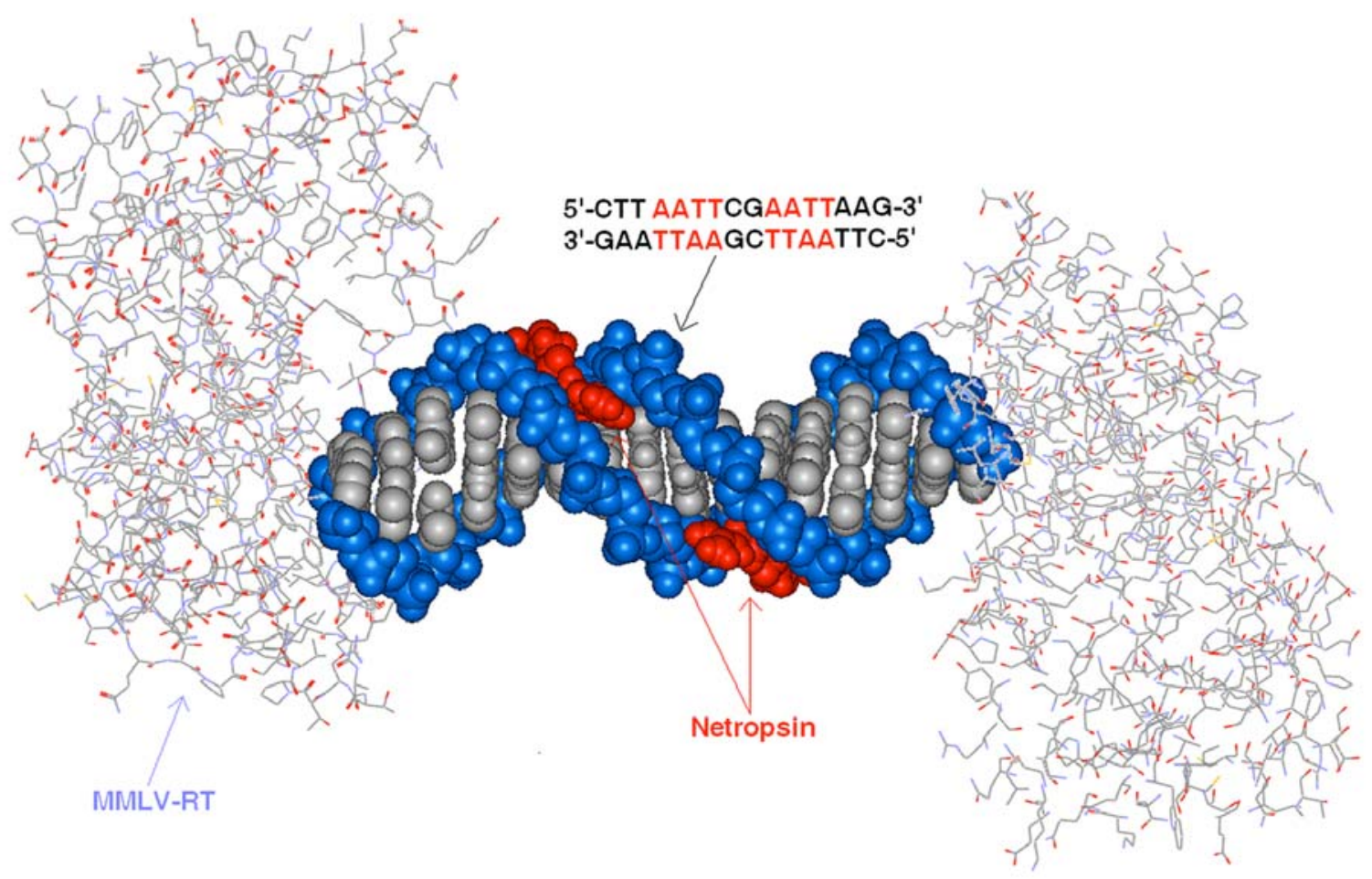

Fig. (1). The crystal structure of two netropsins bound to d(CTTAATTCGAATTAAG) $)_{2}$ in a host-guest complex with the N-terminal fragment of Moloney murine leukemia virus reverse transcriptase (MMLV RT) catalytic fragment [9]. The DNA oligonucleotide is shown in blue and gray CPK model and netropsin is shown in a red CPK model. The host protein fragment (MMLV RT) is shown as a line model colored by atom type. 
constraints were not used. All analyses were carried out using the CARNAL and ANAL programs in AMBER.

\section{Energy Calculations}

Prior to energy calculations, solvent water molecules and counterions were removed from the system. Gas-phase molecular mechanical (MM) energies were subsequently averaged over all snapshots acquired between $0.5 \mathrm{~ns}$ and 10 ns. These energies included contributions from bonded and nonbonded electrostatic and van der Waals interactions for the DNA/netropsin complex, free DNA and free netropsin. All MM calculations were performed with a non-bonded cutoff of $99 \AA$ and a dielectric constant of 1 . For binding energy calculations, the DNA and netropsin geometries were taken from those of the corresponding complex used in the calculation.

\section{RESULTS AND DISCUSSION}

\section{RMS Deviations}

Potential energy stabilization and RMS deviations indicated the attainment of system equilibrium during the simulation. System equilibrium occurred at $250 \mathrm{ps}$ and coordinates were collected every 0.2 ps between 500 ps and 10 ns during the simulation. Panel A of Fig. (2) shows the RMS deviations of DNA in the simulation of the complex
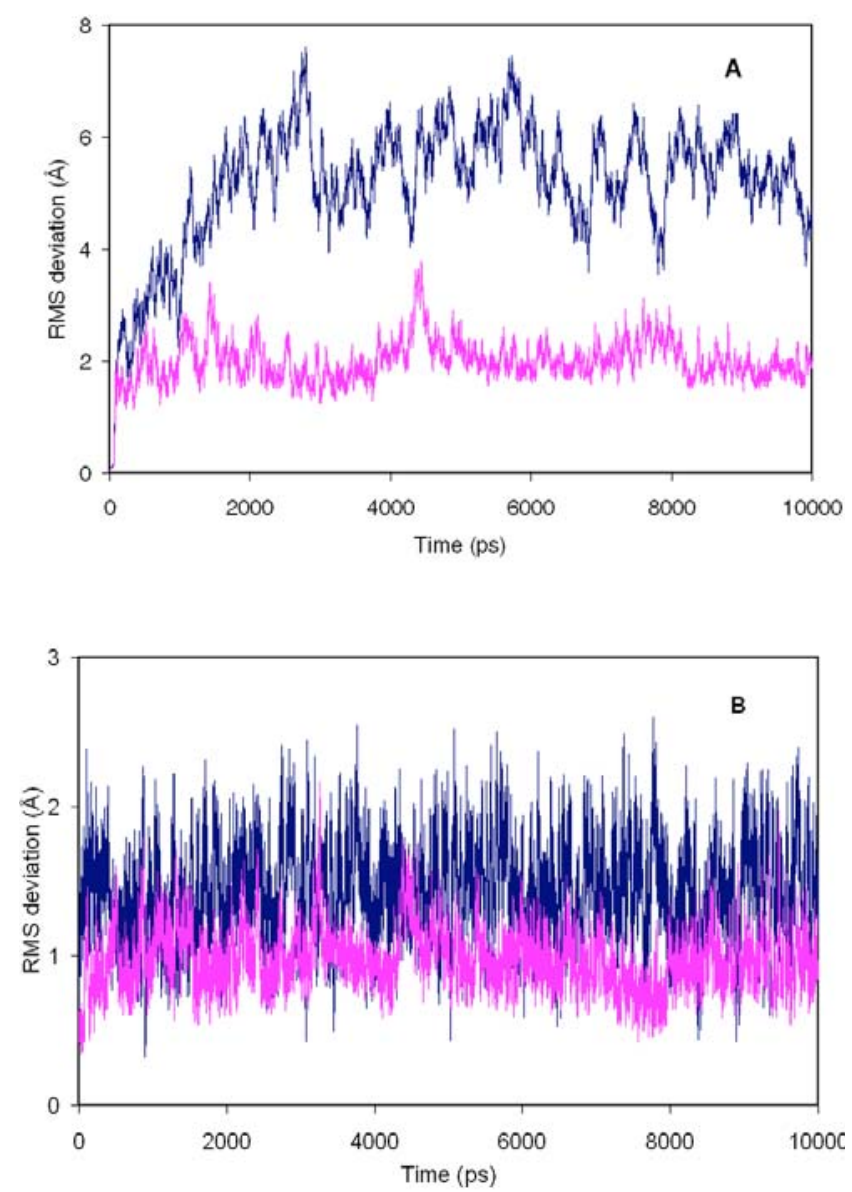

Fig. (2). A. RMS deviations of complexed (pink) and free DNA (blue). B. RMS deviations of complexed (pink) and free (blue) netropsin. (pink) and the simulation of the corresponding uncomplexed or free DNA (blue). Complexed DNA shows a consistently lower average RMS deviation relative to the free DNA (2.05 $\AA$ vs. $5.35 \AA$ ), suggesting increased flexibility of free DNA. Panel B shows the RMS deviations of bound and free netropsin. In this case, the behavior of complexed netropsin is much closer to that of free netropsin, suggesting that when netropsin resides in the DNA minor groove it experiences relatively unhampered motional freedom.

\section{Weighted Average Structures and Netropsin Binding Orientation}

Fig. (3) shows the weighted average structures for the netropsin/DNA complex and free DNA. The weighted average structures were determined from the coordinate sets whose structures were closest to the calculated average structure. As can be seen, two netropsin molecules are bound in the DNA minor groove. Netropsin1 (Nt1) is shown in yellow and the netropsin $2(\mathrm{Nt} 2)$ is shown in green.
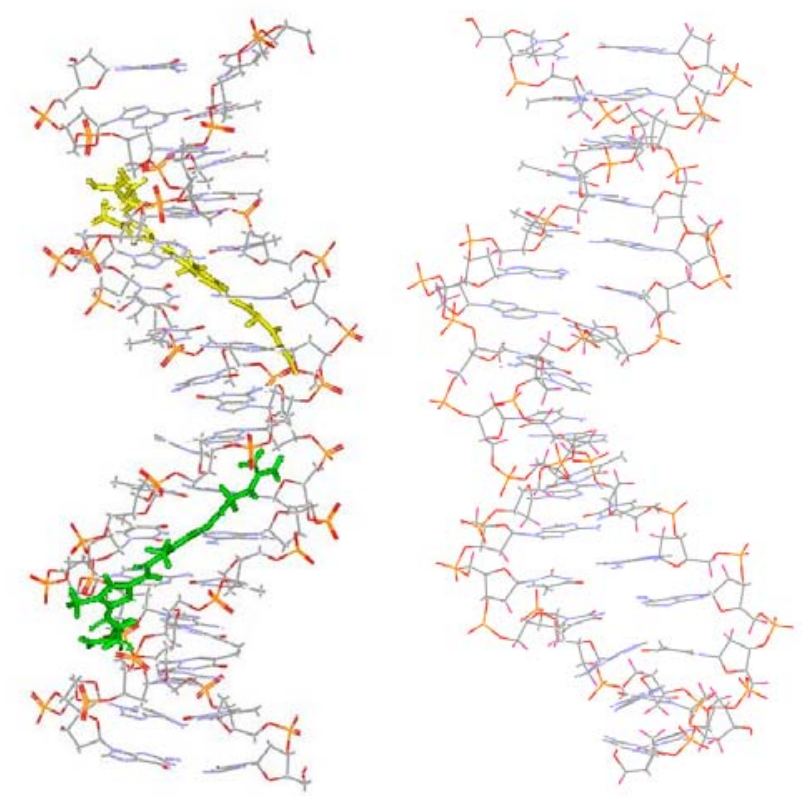

Fig. (3). Average structures of the netropsin/DNA complex (left) and the free DNA (right) from simulations of complex and free DNA. Hydrogen atoms were removed for clarity.

The schematic to the right in Fig. (4) represents the binding orientation of the two netropsins to the DNA. The guanidinium terminus of each netropsin was directed towards the dyad of the oligonucleotide structure in a headto-head orientation. The schematic on the left in Fig. (4) represents the hydrogen-bonding patterns observed during the simulation. During the simulation, any hydrogen bond that existed for more than $5 \%$ of the time is presented as a dashed line. As shown, the patterns of H-bond interactions between DNA and the two netropsins were found to be identical.

\section{Binding Energies}

The binding energies of the two netropsins bound to d(CTTAATTCGAATTAAG) ${ }_{2}$ were calculated using the average potential energies for the complex $\left(E_{\text {Complex }}\right)$, free 

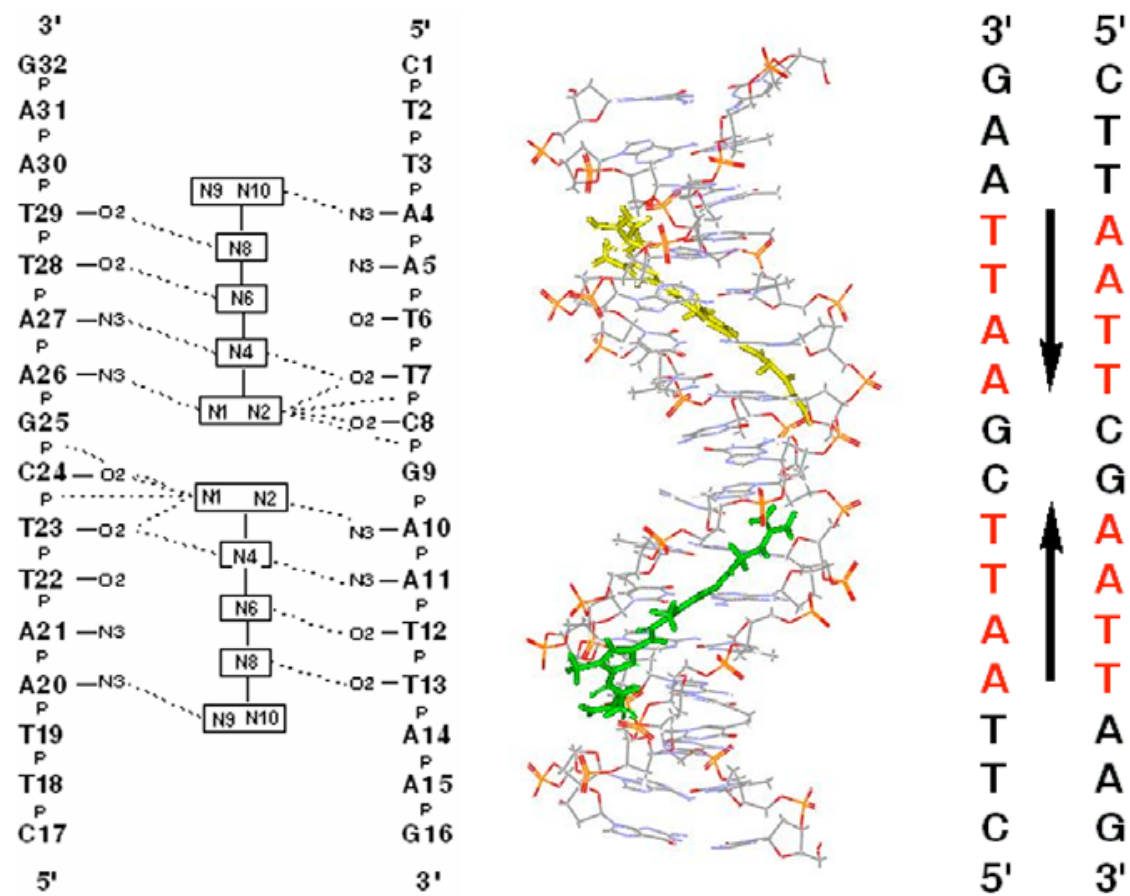

Fig. (4). Hydrogen-bonding patterns (left) and binding orientations (right) of netropsins bound to d(CTTAATTCGAATTAAG) 2 .

DNA $\left(E_{D N A-F r e e}\right)$ and free netropsin $\left(E_{\text {Netropsin-Free }}\right)$. The resulting netropsin binding energy was calculated according to the following equation:

$\Delta E=E_{\text {Complex }}-E_{D N A-F r e e}-2 \times E_{\text {Netropsin-Free }}$

and found to be $-2821.18 \mathrm{kcal}(\mathrm{mol})^{-1}$. This result indicated the increased stability of the complex structure relative to the structures of the free components.

It was of interest to evaluate the binding energy of a second netropsin to the complex when one netropsin has already been bound. These calculations were carried out as indicated by the equations below:

$\Delta E_{N t 2}=E_{\text {Complex }}-E_{D N A-N t 1}-E_{N t 2}$

and
$\Delta E_{N t 1}=E_{C o m p l e x}-E_{D N A-N t 2}-E_{N t 1}$

Results from these evaluations are shown in Table (1). The calculated binding energies of Nt1 and Nt2 are -1365.17

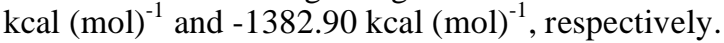

\section{Dynamic Behavior of Netropsin}

Netropsin may be considered to consist of four rigid planes connected by three flexible linkers. The planes are indicated in Scheme $\mathbf{2}$ as the colored areas. During the simulation, these planes may rotate relative to each other, as indicated by the angles 1, 2, and 3 defined in Scheme 2. Fig. (5) shows the rotation of angles 1,2, and 3 observed during the simulation.

These results indicate that angle 3 shows significant flexibility in free netropsin, while both bound netropsins are

Table 1. Netropsin Binding Energies Determined in the Simulation of Two Netropsins Bound to d(CTTAATTCGAATTAAG) ${ }_{2}{ }^{a}$

\begin{tabular}{|c|c|c|c|c|c|c|c|c|}
\hline & \multicolumn{4}{|c|}{ Nt1 } & \multicolumn{4}{|c|}{ Nt2 } \\
\hline 1,4 VDW & 284.39 & 278.29 & 6.10 & & 284.39 & 278.32 & 6.07 & \\
\hline Angle & 615.50 & 583.80 & 31.70 & & 615.50 & 583.67 & 31.83 & \\
\hline Dihedral & 750.00 & 732.41 & 17.59 & & 750.00 & 732.85 & 17.15 & \\
\hline EEL & 2679.12 & 3915.17 & 58.26 & -1294.31 & 2679.12 & 3929.20 & 61.66 & -1311.74 \\
\hline VDW & -704.86 & -633.60 & -0.56 & -70.70 & -704.86 & -663.29 & -0.40 & -41.17 \\
\hline Total & 1690.17 & 3069.46 & -14.28 & -1365.01 & 1690.17 & 3085.78 & -12.71 & -1382.90 \\
\hline
\end{tabular}

${ }^{a}$ Energies are reported in kilocalories per mole. ${ }^{\mathrm{b}} E_{\text {complex }}, E_{D N A}$, and $E_{\text {ligand }}$ denote the molecular mechanical (MM) energies for the complex, DNA and ligand respectively. ${ }^{\mathrm{c}}$ relative binding energies, calculated by $\Delta E=E_{\text {complex }}-E_{D N A^{-}} E_{\text {ligand }}$. 

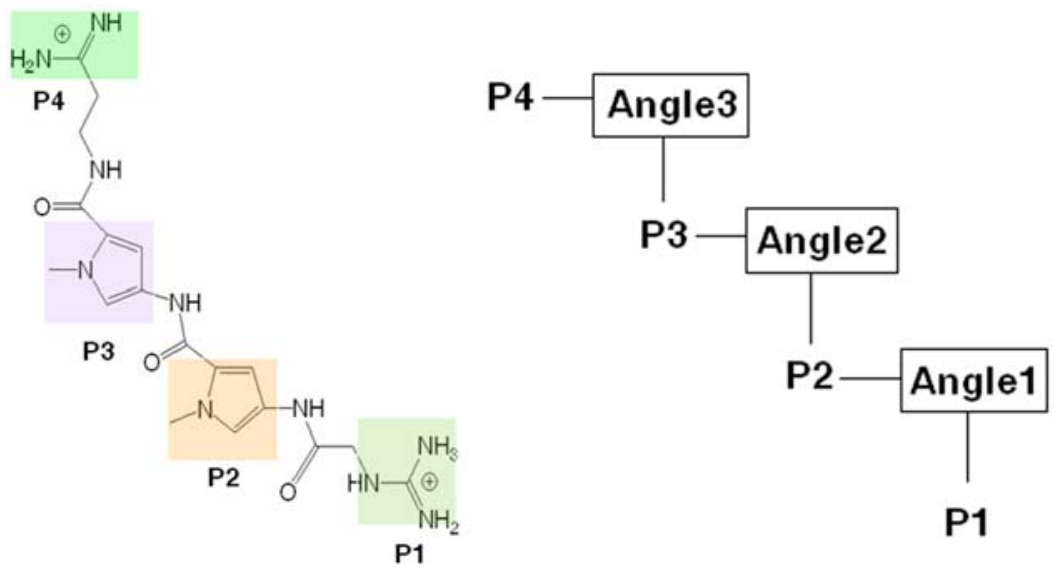

Scheme 2. Rotational planes in netropsin.
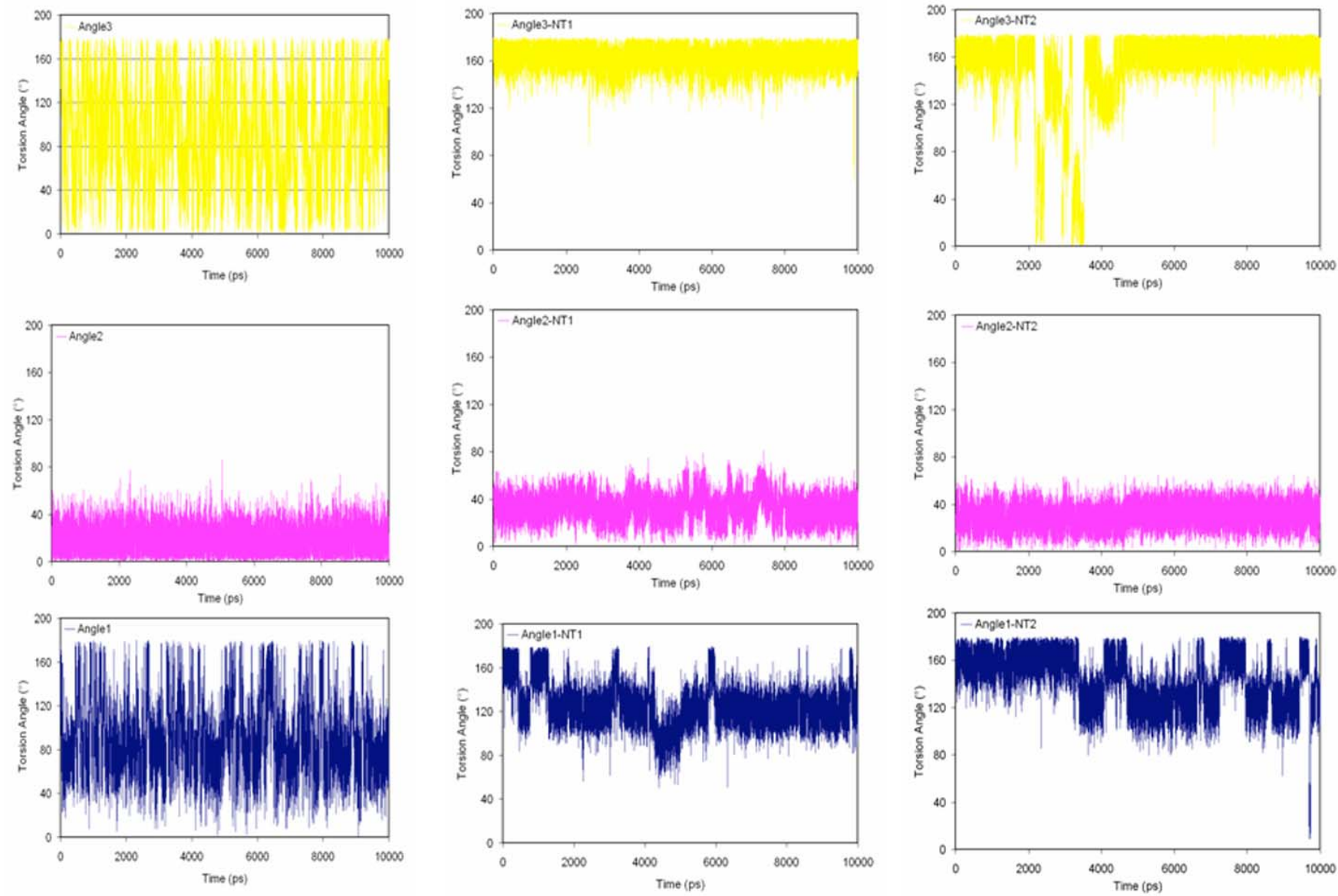

Fig. (5). The left (free netropsin), middle (DNA-bound Nt1), and right (DNA-bound Nt2) columns show the rotations of angle 1 (bottom row), 2 (middle row), and 3 (top row), respectively.

much less flexible. This suggests that the DNA site provides some constraint to this portion of the bound netropsin molecule. Angle 2 shows rigidity in both the free and the bound netropsins, suggesting that this region of the molecule is naturally rigid independent of its bound state; this further suggests an element of ligand structural pre-organization that may assist in DNA binding. Free netropsin shows significant movement in angle 1 while the bound netropsins are again much less mobile as observed for angle 3. However, different from angle 3 , the motion that is observed in angle 1 suggests a patterned sampling of space by angle 1 . Additionally, the pattern of motion in Nt1 is different from that observed in $\mathrm{Nt} 2$. Analysis of the simulation trajectories shown in Fig. (6) indicated that the guanidinium terminus of $\mathrm{Nt} 2$ is often in a downward folded conformation and the 

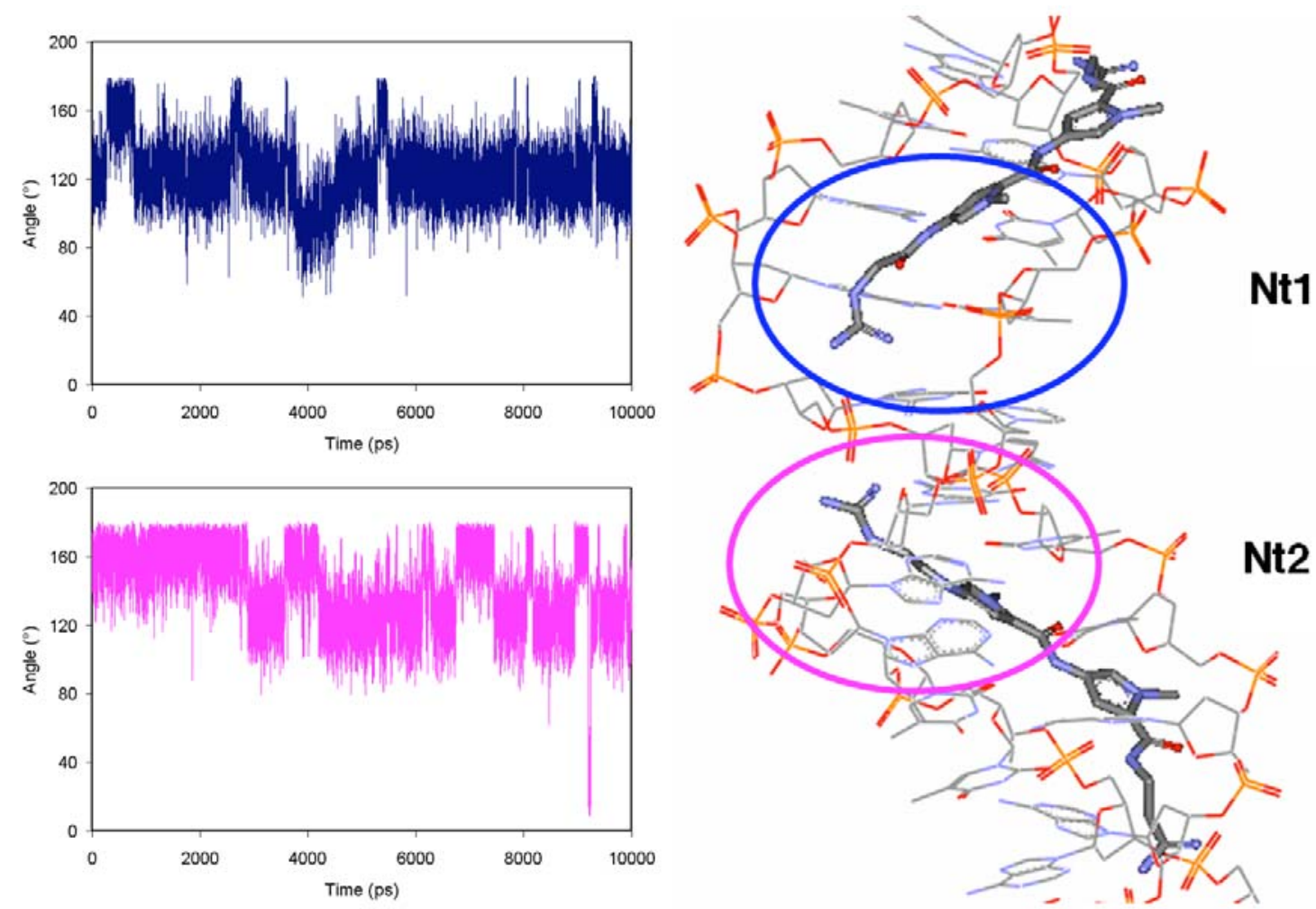

Fig. (6). Dynamic behavior of the guanidinium termini of Nt1 and Nt2 (left) and a typical structure with bent conformation of Nt1 and extended conformation of $\mathrm{Nt} 2$ at their guanidinium termini, respectively.

guanidinium terminus of $\mathrm{Nt} 1$ is in a more extended conformation.

\section{Minor Groove Width Fluctuations}

As shown in Fig. (7), the width of the minor groove is defined as the closest distance between phosphates along the duplex strand. The schematic on the right in Fig. (8) illustrates minor groove widths where the guanidinium (pink) and amidinium (blue) termini of the two netropsins are located. The fluctuation of the minor groove width in these areas is shown in the plots on the left of Fig. (8). As can be seen, the amidinium and guanidinium termini of the two bound netropsins behave differently during the simulation. Particularly, in the case of $\mathrm{Nt} 1$ there seems to be some patterned behavior in minor groove width changes, indicated by a decrease in angle 1 around 4000 ps. In the case of $\mathrm{Nt} 2$ the changes are more subtle, but the differences in the minor groove width at the two termini of the netropsin appear to diverge as the simulation proceeds.

The X-ray study [9] found that the minor groove width contributes to the orientation of a bound netropsin with the guanidium terminus bound to the narrower part in the center of the sequence and the amidinium terminus bound to the wider part at the end of the sequence. Our molecular dynamics simulation results are thus in good agreement with these experimental results. Moreover, it should be noted that while the two netropsins are bound to identical sites in the palindromic DNA, their dynamic behaviors differ. Therefore, it appears that small molecule recognition and binding by DNA may be governed by mechanism(s) that are much more complex than initially anticipated and may represent unexpected challenges in genome-targeted drug design that go well beyond simple sequence readout.

\section{CONCLUSIONS}

Three $10 \mathrm{~ns}$ molecular dynamics simulations of a unique netropsin/DNA co-complex, relevant free DNA, and free

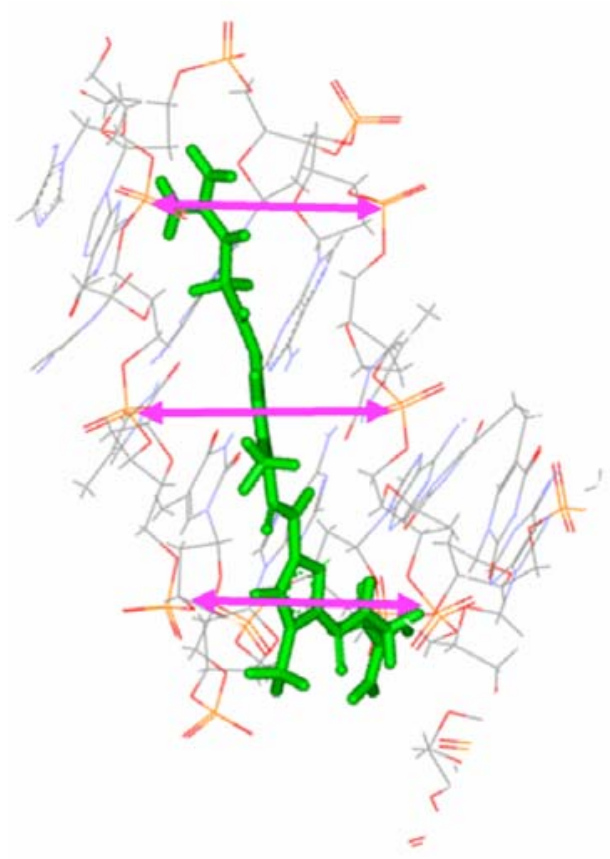

Fig. (7). The width of DNA minor groove is defined (arrows) as the closest distance between two phosphates. 

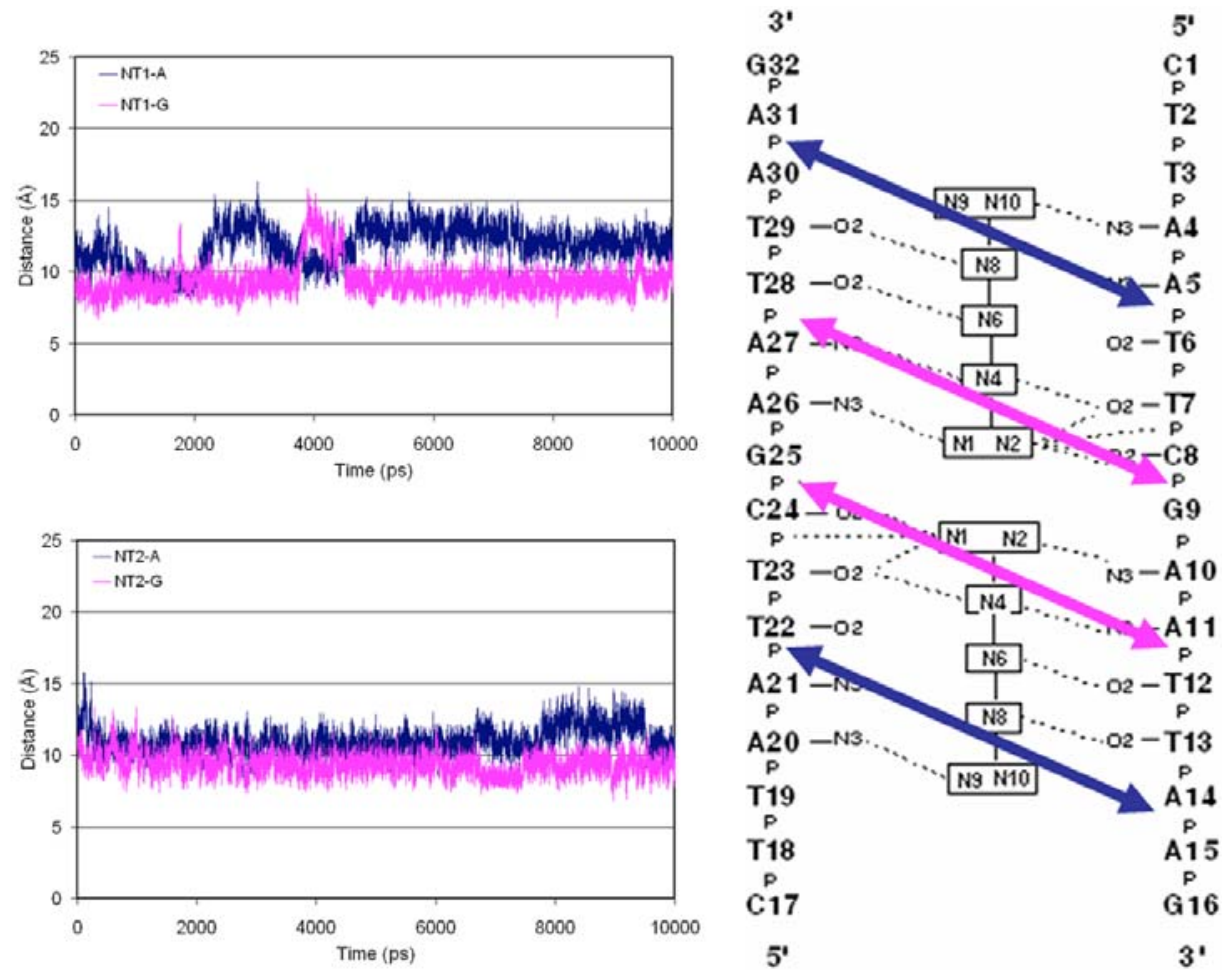

Fig. (8). The arrow lines on the right show the definition of the width of the minor groove at two places where the guanidinium and amidinium termini of the two bound netropsins are located. The plots to the left show the minor groove width fluctuation at the guanidinium and amidinium termini of netropsin during the simulation.

netropsin were carried out in order to investigate factors responsible for netropsin binding to DNA. Surprisingly, our results indicated that the two netropsins, bound to a single DNA duplex, exhibited differences in their dynamic performances, including differences in their RMS deviations, binding energies, minor groove width fluctuations, and rotations of their structural planes, respectively. These observations suggest that the dynamic performance of small molecules and DNA in their respective DNA bound complexes may have a greater impact on small molecule DNA recognition than realized, indicating unexpected challenges in genome-targeted drug design.

\section{ACKNOWLEDGEMENT}

This work is supported by grant GM 62831 from NIH (to E.C.L.) and 2 G12 RR003048 from the RCMI Program, Division of Research Infrastructure, National Center for Research Resources, NIH.

\section{REFERENCES}

[1] Dickinson, L.A.; Burnett, R.; Melander, C.; Edelson, B.S.; Arora, P.S.; Dervan, P.B.; Gottesfeld, J.M. Arresting cancer proliferation by small-molecule gene regulation. Chem. Biol., 2004, 11, 15831594.

[2] Neidle, S. DNA minor-groove recognition by small molecules. Nat. Prod. Rep., 2001, 18, 291-309.
[3] Sriram, M.; van der Marel, G.A.; Roelen, H.L.; van Boom, J.H.; Wang, A.H.J. Structural consequences of a carcinogenic alkylation lesion on DNA: Effect of O6-ethylguanine on the molecular structure of the d(CGC[e6G]AATTCGCG)- netropsin complex. Biochemistry, 1992, 31, 11823-11834.

[4] Balendiran, K.; Rao, S.T.; Sekharudu, C.Y.; Zon, G.; Sundaralingam, M. X-ray structures of the B-DNA dodecamer d(CGCGTTAACGCG) with an inverted central tetranucleotide and its netropsin complex. Acta Crystallogr., Sect. D., 1995, 51, 190 198.

[5] Lown, J.W. DNA recognition by lexitropsins, minor groove binding agents. J. Mol. Recognit., 1994, 7, 79-88.

[6] Dervan, P.B.; Edelson, B.S. Recognition of the DNA minor groove by pyrrole-imidazole polyamides. Curr. Opin. Struct. Biol., 2003, 13, 284-299.

[7] Wellenzohn, B.; Winger, R.H.; Hallbrucker, A.; Mayer, E.; Liedl, K.R. Simulation of EcoRI dodecamer netropsin complex confirms class I complexation mode. J. Am. Chem. Soc., 2000, 122, 3927 3931.

[8] Wellenzohn, B.; Flader, W.; Winger, R.H.; Hallbrucker, A.; Mayer, E.; Liedl, K.R. Significance of ligand tails for interaction with the minor groove of B-DNA. Biophys. J., 2001, 81, 1588 - 1599.

[9] Goodwin, K.D.; Long, E.C.; Georgiadis, M.M. A host-guest approach for determining drug-DNA interactions: An example using netropsin. Nucleic Acids Res., 2005, 33, 4106 - 4116.

[10] Sun, D.; Jessen, S.; Liu, C.; Liu, X.; Najmudin, S.; Georgiadis, M.M. Cloning, expression, and purification of a catalytic fragment of Moloney murine leukemia virus reverse transcriptase: Crystallization of nucleic acid complexes. Protein Sci., 1998, 7, $1575-1582$

(C) Fang et al.; Licensee Bentham Open.

This is an open access article licensed under the terms of the Creative Commons Attribution Non-Commercial License (http://creativecommons.org/licenses/by-nc/3.0/) which permits unrestricted, non-commercial use, distribution and reproduction in any medium, provided the work is properly cited. 